\title{
Toward Minimally Invasive Therapeutic Ultrasound: Ultrasound-guided Ablation in Neuro-oncology
}

Micah Belzberg ${ }^{1}$, Smruti Mahapatra ${ }^{2}$ Francisco Chavez ${ }^{3}$, Kyle Morrison ${ }^{3}$, Kah Timothy Xiong ${ }^{3}$, Nao J. Gamo ${ }^{4}$, Stephen Restaino ${ }^{4}$, Rajiv Iyer $^{5}$, Mari Groves ${ }^{5}$, Nitish Thakor ${ }^{2}$, Nicholas Theodore ${ }^{2,5}$, Mark G. Luciano ${ }^{2,5}$, Henry Brem², ${ }^{2,5}$ Alan R. Cohen ${ }^{5}$, Amir Manbachi ${ }^{2,5}$

${ }^{1}$ Department of Plastic Surgery, Johns Hopkins University, Baltimore, MD

${ }^{2}$ Department of Biomedical Engineering, Johns Hopkins University, Baltimore, MD

${ }^{3}$ Sonic Concepts, Inc., Bothell, WA

${ }^{4}$ Maryland Development Center, Baltimore, MD

${ }^{5}$ Department of Neurosurgery, Johns Hopkins University, Baltimore, MD

\section{Corresponding Author's Address:}

Amir Manbachi, Ph.D.

Departments of Neurosurgery and Biomedical Engineering,

Johns Hopkins University

600 N. Wolfe St., Meyer 8-181C

Baltimore, MD 21287, USA

Email: Amir.Manbachi@jhu.edu

Funding: This study was funded by Maryland Technology Development Corporation's Maryland Innovation Initiative, Coulter Foundation, National Science Foundation's I-Corps program and Johns Hopkins University, Whiting School of Engineering's Cohen Translational Funding opportunities.

Financial Disclosures: Kyle Morrison, Francisco Chavez, and Kah Timothy Xiong are employees of Sonic Concepts, Inc. Dr. Brem is a consultant serving as Medical Advisory Board Chairman for InSightec. He is also on the board of directors for Galen Robotics. Dr. Nao J Gamo is the founder and CEO of Neurosonics Medical, Inc. Dr. Stephen Restaino is the Director of Engineering at Maryland Development Center, a startup studio supporting local medical device innovations.

Acknowledgments: The authors would like to thank Sonic Concepts, Inc. (Bothell, Washington) for manufacturing the custom probe, as well as Maryland Development Center and Dr. Gil Blankenship for support in software development. NICO Corporation's Joe Mark and Michele Kennedy are acknowledged for providing their minimally invasive BrainPath ${ }^{\mathrm{TM}}$ product for free. 


\section{ABSTRACT (<250 words, Currently 249)}

2 Introduction: To improve patient outcomes (eg, reducing blood loss and infection), practitioners

3 have gravitated toward noninvasive and minimally invasive surgeries (MIS), which demand

4 specialized toolkits. Focused ultrasound, for example, facilitates thermal ablation from a distance,

5 thereby reducing injury to surrounding tissue. Focused ultrasound can often be performed

6 noninvasively; however, it is more difficult to carry out in neuro-oncological tumors, as ultrasound

7 is dramatically attenuated while propagating through the skull. This shortcoming has prompted

8 exploration of MIS options for intracranial placement of focused ultrasound probes, such as within

9 the BrainPath ${ }^{\mathrm{TM}}$ (NICO Corporation, Indianapolis, IN). Herein, we present the design,

10 development, and in vitro testing of an image-guided, focused ultrasound prototype designed for

11 use in MIS procedures. This probe can ablate neuro-oncological lesions despite its small size.

12 Materials \& Methods: Preliminary prototypes were iteratively designed, built, and tested. The

13 final prototype consisted of three 8-mm-diameter therapeutic elements guided by an imaging

14 probe. Probe functionality was validated on a series of tissue-mimicking phantoms.

15 Results: Lesions were created in tissue-mimicking phantoms with average dimensions of

$162.5 \times 1.2 \times 6.5 \mathrm{~mm}$ and $3.4 \times 3.25 \times 9.36 \mathrm{~mm}$ after 10 - and 30 -second sonification, respectively. $30 \mathrm{~s}$

17 sonification with $118 \mathrm{~W}$ power at $50 \%$ duty cycle generated a peak temperature of $68^{\circ} \mathrm{C}$. Each

18 ablation was visualized in real time by the built-in imaging probe.

19 Conclusion: We developed and validated an ultrasound-guided focused ultrasound probe for use

20 in MIS procedures. The dimensional constraints of the prototype were designed to reflect those of

21 BrainPath trocars, which are MIS tools used to create atraumatic access to deep-seated brain

22 pathologies. 


\section{$1 \quad$ KEYWORDS $(<\mathbf{1 0})$}

2 Ultrasound, Therapeutic Ultrasound, Focused Ultrasound, Ablation, Engineering Design,

3 Transducer Design, Acoustic Probes, Minimally Invasive Surgery, Neurosurgery, Oncology

5 HIGHLIGHTS (core findings, 3-5 bullets (max 85 characters including spaces, per bullet

6 point).

- An ultrasound-guided, focused ultrasound prototype was developed and validated

- The therapeutic transducer $(1.5 \mathrm{MHz})$ consisted of three $8-\mathrm{mm}$ circular elements

- Elements were placed on a 9x32mm curved rectangular aperture: $45 \mathrm{~mm}$ radius curvature

11 - Functionality was examined on tissue-mimicking phantoms 


\section{1. INTRODUCTION}

Focused ultrasound is an appealing tool for use in both noninvasive and minimally invasive

3 surgical (MIS) procedures, as it can ablate pathologic tissue from a distance. ${ }^{1-3}$ This is particularly

4 helpful in patients diagnosed with inoperable tumors. ${ }^{4,5}$ In neurosurgery, noninvasive

5 (transcranial) focused ultrasound helmet systems have been successfully used to treat movement

6 disorders, including Parkinson disease and essential tremor. ${ }^{6}$ However, their use for ablation of

7 neuro-oncological tumors faces certain drawbacks; ${ }^{3}$ namely, significant attenuation of the

8 ultrasonic wave during propagation in the skull, which requires high ultrasonic powers directed at

9 the patient's head. ${ }^{7-9}$ In response to these drawbacks, MIS focused ultrasound approaches have

10 been contemplated, as they may require only miniature probes to ablate intracranial lesions from

11 within the cranium. ${ }^{10,11}$

One such example is a minimally invasive, focused ultrasound probe capable of being

13 placed within the BrainPath ${ }^{\mathrm{TM}}$ (NICO Corporation, Indianapolis, IN), a device that allows

14 atraumatic access to brain oncology (eg, deep-seated tumors) in MIS settings (Fig. 1). The

15 development of products like BrainPath ${ }^{\mathrm{TM}}$ represents a growing trend toward MIS options in

16 healthcare, where surgical interventions are increasingly being performed with minimally invasive

17 approaches or keyhole procedures to enhance patient outcomes and reduce blood loss or

18 infection. $^{12}$ Of course, this trend requires development of novel, precise, miniature surgical

19 instruments. Although larger transducer surface areas allow for better energy deposition, MIS

20 encourages increasingly smaller probes to reduce incision sizes and dissection requirements - and

21 this tradeoff demands better understanding of acoustic designs to balance both criteria. ${ }^{13,14,15}$ In

22 this study, we investigated preliminary focused ultrasound configurations for use in MIS settings,

23 particularly for use in ablation of neuro-oncological lesions. Here, we report the development and 
1 in vitro testing of an initial series of probe designs small enough for use in MIS, yet with a large

2 enough transducer surface area to generate the focal point ablations.

\section{2. MATERIALS AND METHODS}

Therapeutic Elements: Based on the dimensions of BrainPath ${ }^{\mathrm{TM}}$ and the guidelines for MIS

5 and neuroendoscopy techniques (ie, burr holes measuring $18 \mathrm{~mm}$ or smaller), the focused

6 ultrasound transducer was designed to fit within a rectangular aperture of $9 \times 32 \mathrm{~mm}\left(\right.$ Figs. 1-2). ${ }^{16-}$

$7{ }^{18} \mathrm{In}$ order to accommodate the anatomy of a typical adult brain, where a tumor may be 4 to $5 \mathrm{~cm}$

8 away from the probe placement within the BrainPath ${ }^{\mathrm{TM}}$, a transducer with a $45-\mathrm{mm}$ radius of

9 curvature $(\mathrm{RoC})$ was designed to target a natural focal point lesion 4 to $5 \mathrm{~cm}$ away. Building upon

10 our prior simulation studies that investigated the effects of variations in transducer frequency, RoC,

11 and power on the thermal dose and energy deposition in tissue, we developed and manufactured 2

12 alpha prototype transducer designs with center frequency of $1.5 \mathrm{MHz}$ (full width half maximum

13 bandwidth: 1.20-1.80MHz) for in vitro validation (Fig. 2). ${ }^{19,20}$ Design I was a 1-piece, 9x32mm,

14 cylindrically curved rectangular aperture with $45 \mathrm{~mm}$ RoC. Design II contained three 8-mm-

15 diameter circular elements placed on the curved geometry described above. The therapeutic array

16 had a width of $12 \mathrm{~mm}$ casing, which widened to $15 \mathrm{~mm}$ to accommodate a built-in imaging array,

17 as described below.

18 Imaging Elements: To reduce the need for intraoperative magnetic resonance imaging

19 (MRI) guidance, a built-in ultrasound imaging probe was designed to demonstrate proof-of-

20 concept for the ultrasound-guided focused ultrasound (USgFUS) approach studied here. An "off-

21 the-shelf" IP-105 linear imaging probe from Sonic Concepts (Bothell, WA) was chosen. This 64-

22 element, one-dimensional phased array (center frequency: 5.0MHz) was placed within the device

23 housing and was tilted $30^{\circ}$ to provide real-time visualization of the ablation lesion. Customized 
software was developed to drive the probe and to store, study, and modify signals. This software

system (Verasonics Inc, Kirkland, WA).
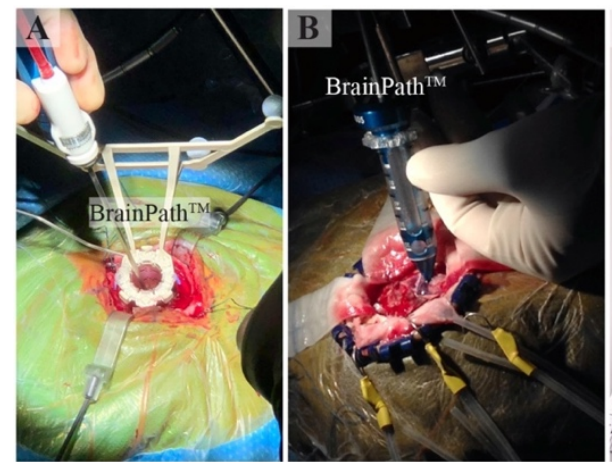

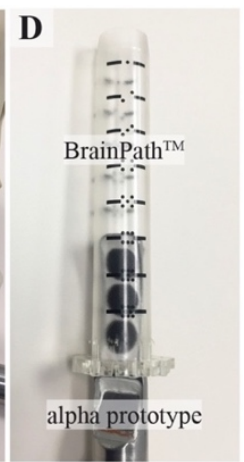

Fig. 1. The BrainPath ${ }^{\mathrm{TM}}$ is a minimally invasive neurosurgical toolkit that can accommodate neuroendoscopic devices. (A\&B) The BrainPath ${ }^{\mathrm{TM}}$ trocar is inserted into brain tissue to create atraumatic surgical access to deep-seated lesions. (C\&D) The ultrasound-guided focused ultrasound alpha prototype probe described in this study is shown in relation to the BrainPath ${ }^{\mathrm{TM}}$ dimensions. A and B contain original images presented with permission from NICO Corporation.

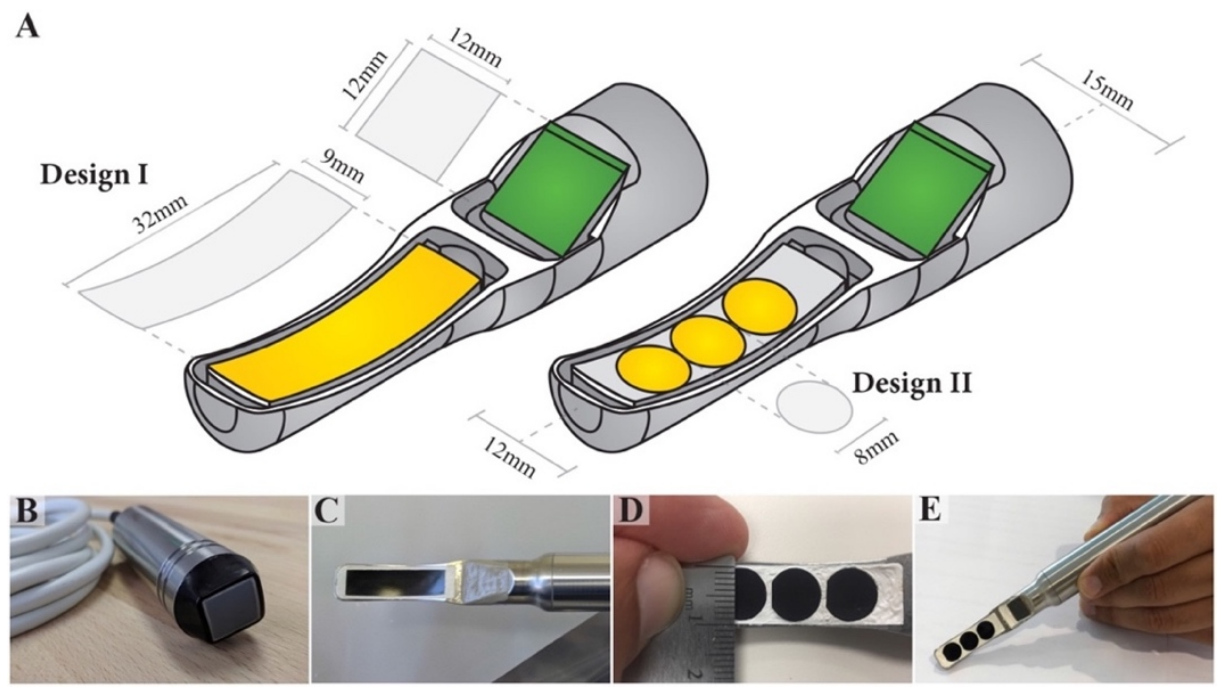

Fig. 2. Probe designs and prototyping details. (A) Two late-stage designs of the custom probe containing a $12 \times 12-\mathrm{mm}$ imaging transducer adjacent to a therapeutic component. Design I is a 1piece, 9x32mm, cylindrically curved rectangular aperture with $45 \mathrm{~mm}$ RoC. Design II contains 3 circular therapeutic elements measuring $8 \mathrm{~mm}$ in diameter, arrayed along a 9x32mm, cylindrically curved rectangular aperture with $45 \mathrm{~mm}$ RoC. The therapeutic array fits within a casing $12 \mathrm{~mm}$ in diameter, which widened to $15 \mathrm{~mm}$ to accommodate the built-in imaging array. (B) A commercial imaging probe (Sonic Concepts IP-105, center frequency: $5 \mathrm{MHz}$ ) which was integrated into our custom ultrasound-guided probe; (C) functional prototype of the custom probe (Design I) housed in a stainless steel casing; (D) 8-mm-diameter circular therapeutic elements (Design II); (E) 

submerging the device in a 4-gallon test tank filled with tap water. Water was degassed to reduce the amount of dissolved gasses within the tank. Water temperature throughout experimental testing was $23^{\circ} \mathrm{C}$, with less than $1^{\circ} \mathrm{C}$ variation. An acoustic absorber was placed inside the tank to reduce acoustic reflections from the tank edges. A calibrated oscilloscope as well as voltage and current

6 probes were used to measure the voltage, current, and subsequently the net power of the device under test conditions. Using a three-dimensional (3D) printed holder created on a commercial 3D

8 printer (Objet260, Connex3, Stratasys Ltd., Eden Prairie, MN), blocks of solid water phantoms were positioned at the focal point. Solid water is a tissue-mimicking phantom, proprietary to Sonic

Concepts, with acoustic properties (ie, attenuation and propagation velocity) similar to those of

11 water. The absorption coefficient at $1 \mathrm{MHz}$ is $\sim 0.01 \mathrm{~dB} / \mathrm{cm}$. Sound speed is $1,500 \mathrm{~m} / \mathrm{s}$. The

12 phantoms were used to create predictable lesions and correlate results with calibrated hydrophone

13 measurements. Lesion generation was observed both visually and via the built-in imaging probe

14 driven by a Vantage 64 LE system (Verasonics, Inc., Kirkland, WA). The experimental design is

15 shown in Fig. 3.

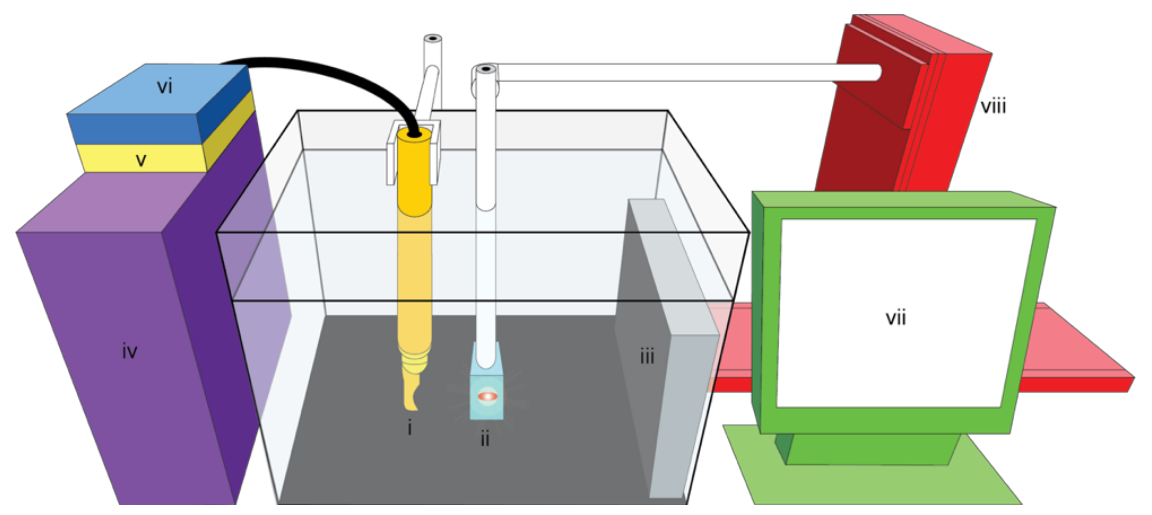

17 Fig. 3. Experimental setup. (i) ultrasound-guided focused ultrasound prototype, (ii) tissuemimicking phantom, (iii) acoustic absorber, (iv) Vantage 64 LE system, (v) pre-amplifier, (vi) matching network, (vii) screen for real-time monitoring of ablation, and (viii) stepper motor to raise solid water tissue-mimicking phantoms with each sonification. 
A $1.620-\mathrm{MHz}$ sonification waveform with a pulse period of $10 \mathrm{~ms}$ and $50 \%$ duty cycle was generated. The solid water sample was sonicated for $10 \mathrm{~s}$. To test the built-in imaging probe of the

3 device, brightness-mode (B mode) images were recorded using the imaging transducer described 4 above.

The solid water sample was raised $5 \mathrm{~mm}$ parallel to the long shaft of the device and, using

6 the aforementioned parameters, an additional lesion was created. This process was repeated to create 5 lesions (Fig. 4). During each ablation, ultrasound images were recorded from the built-in

8 imaging probe of the device. A new solid water block was loaded, and the above-described 9 procedure was repeated; however, a 30s sonification was now performed for 3 trials. Axial, lateral, and elevation dimensions of the lesions were measured using a slide micrometer under microscope.

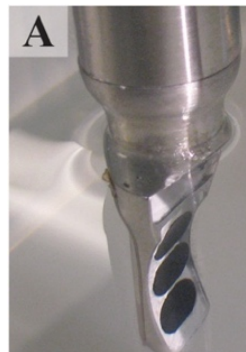

alpha prototype

3D printed holder for tissue mimicking phantom

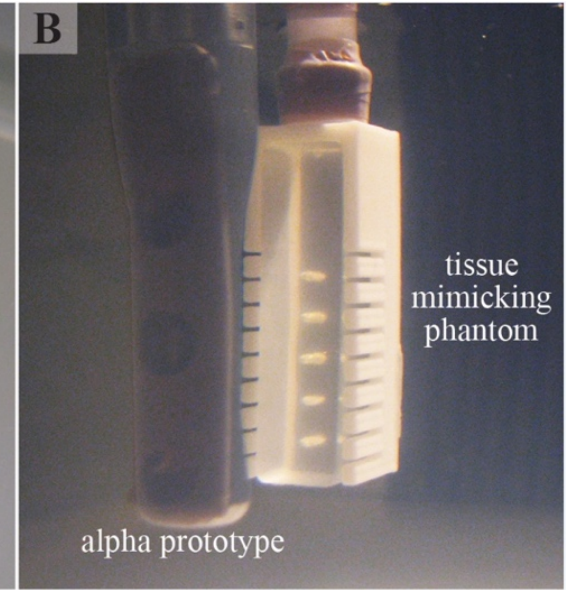

12 Fig. 4. Validation of the prototype. (A) Device aligned with the 3D-printed holder. (B) The 13 prototype generated lesions in tissue-mimicking phantoms secured within the 3D-printed holder.

14 Subsequently, another block was placed in the water-filled tank at the acoustic maximum, 15 as experimentally detected by a hydrophone (Y-Series High Intensity Hydrophones, Sonic 16 Concepts Inc.). A thermocouple was assembled using a Type T thermocouple connector (SMPW17 TM, OMEGA Engineering, Karvina, Czech Republic) and a 40-gauge Type T thermocouple wire 18 with formvar enamel insulation (Pelican Wire, Naples, FL). 
The thermocouple was inserted into the block at the acoustic maximum. Temperature measurements were acquired using a microprocessor thermometer (HH23, OMEGA Engineering).

3 Per the aforementioned parameters, a 30s sonification was performed with continuous temperature

4 measurements. As mentioned previously, peak power was applied at 50\% duty cycle.

\section{RESULTS}

A prototype device based on Design I (Fig. 2) was built, but the design was abandoned due to lateral mode effects that resulted in multiple unwanted focal points and overheating during

8 initial trials. An alpha prototype based on Design II was developed and underwent the above-

9 described testing. At $118 \mathrm{~W}$ and 50\% duty cycle, Design II successfully generated lesions in the

10 tissue-mimicking phantoms (Fig. 5 \& Supplementary Video 1). Sonification for a duration of 10s

11 resulted in lesions with average

12 dimensions of $2.5 \times 1.2 \times 6.5 \mathrm{~mm}$ (lateral,

13 elevation, and axial alignments relative to

14 the transducer). Additionally, lesions

15 created with 30 s sonifications resulted in

16 average dimensions of $3.4 \times 3.25 \times 9.36 \mathrm{~mm}$

17 (Table 1).

Fig. 5. Results of 10 - and 30 -second ablation of solid water samples. The maximum linear dimension of each lesion created was measured using a slide micrometer under microscope.
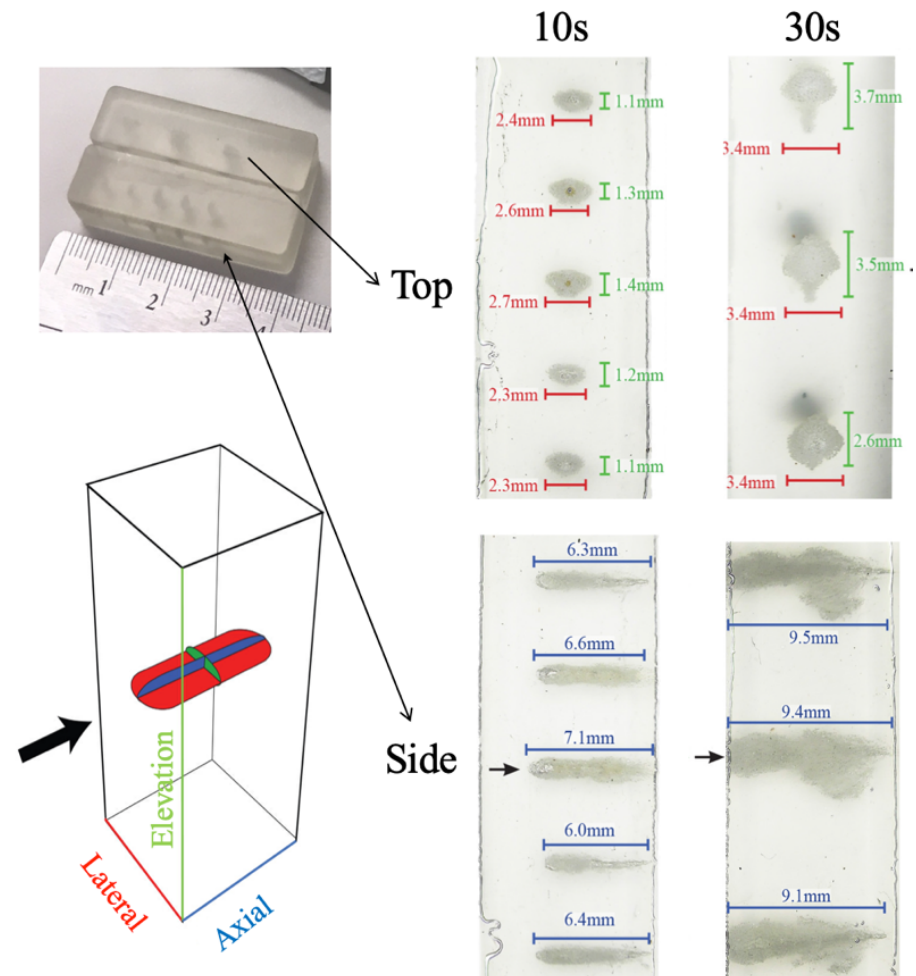
$1 \quad$ Video 2). Precise ablation was visualized by the built-in imaging probe during both $10 \mathrm{~s}$ and $30 \mathrm{~s}$

2 sonifications of the phantom blocks.

6

Table 1: Ablation measurements. *SD, standard deviation.

\begin{tabular}{|l|ccc|ccc|}
\hline & \multicolumn{3}{|c|}{ 10s sonification } & \multicolumn{3}{c|}{ 30s sonification } \\
\hline Axis & Z (mm) & Y (mm) & X (mm) & Z (mm) & Y (mm) & X (mm) \\
\hline Trial & & & & & & \\
$\mathbf{1}$ & 2.29 & 1.09 & 6.45 & 3.39 & 3.71 & 9.53 \\
$\mathbf{2}$ & 2.33 & 1.19 & 6.01 & 3.42 & 3.50 & 9.43 \\
$\mathbf{3}$ & 2.71 & 1.43 & 7.11 & 3.40 & 2.56 & 9.15 \\
$\mathbf{4}$ & 2.60 & 1.29 & 6.63 & & & \\
$\mathbf{5}$ & 2.36 & 1.08 & 6.32 & & & \\
Average & 2.46 & 1.22 & 6.50 & 3.40 & 3.26 & 9.37 \\
Min & 2.29 & 1.08 & 6.01 & 3.39 & 2.56 & 9.15 \\
Max & 2.71 & 1.43 & 7.11 & 3.42 & 3.71 & 9.53 \\
SD* & 0.19 & 0.15 & 0.41 & 0.01 & 0.61 & 0.20 \\
\hline
\end{tabular}

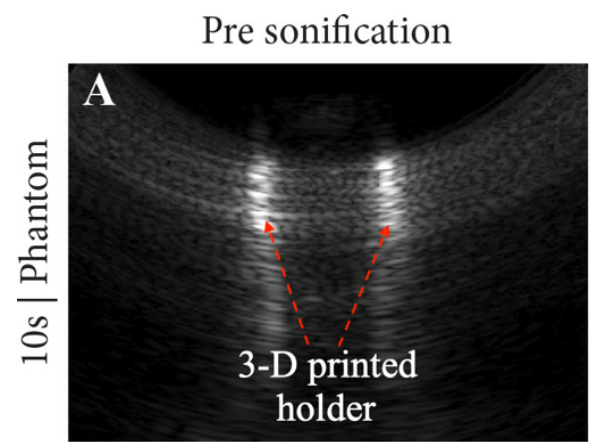

Post sonification

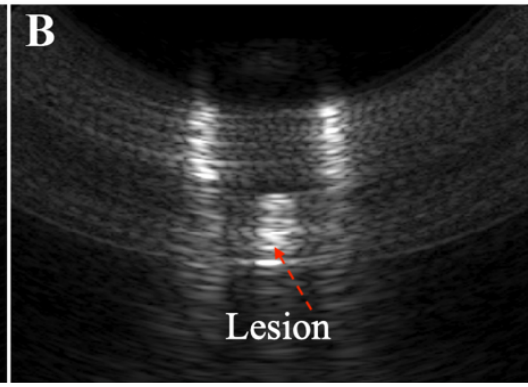

Difference

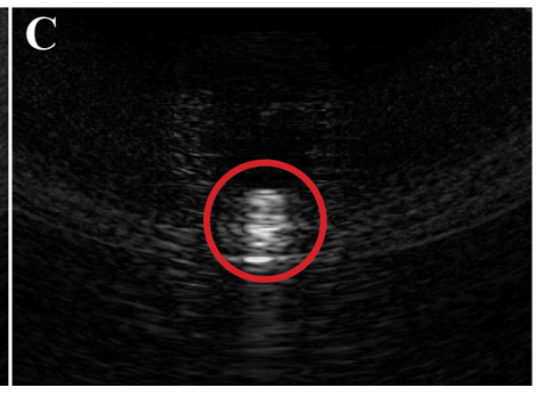

Fig. 6. 10s sonification of solid water phantom as visualized by the built-in imaging probe: (A) pre ablation, (B) post ablation, and (C) the difference between. The red circle outlines the lesion.

\section{DISCUSSION}

Focused ultrasound devices facilitate targeted tissue ablation from a distance, providing a means to treat pathology previously considered inoperable due to inaccessibility. Noninvasive, transcranial focused ultrasound is limited by the acoustic properties of skull bone., ${ }^{2,3}$ Minimally invasive, intracranial focused ultrasound circumvents these limitations, particularly in patients with excessively dense skulls. ${ }^{21}$ However, developing focused ultrasound devices for use in MIS 
1 is challenging. Although acoustic physics favors larger transducer surface areas to achieve

2 ablation, MIS demands tools with increasingly smaller dimensions. ${ }^{13,14,15}$ The optimal geometry

3 for functional, miniature focused ultrasound devices is not well studied. ${ }^{13}$ Therefore, continued

4 size reduction of focused ultrasound transducers requires further investigation of probe acoustic

5 designs (ie, dimensions, curvatures, number of elements, etc.) that can achieve tissue ablation

6 while meeting clinical requirements. This study summarizes a first step in the development of a

7 focused ultrasound device small enough for use in MIS procedures such as neuro-oncological

8 treatment performed with BrainPath ${ }^{\mathrm{TM}}$. We have shown that a focused ultrasound device

9 consisting of three 8-mm-diameter circular therapeutic elements arrayed along a 9x32mm

10 cylindrically curved rectangular aperture with $45 \mathrm{~mm} \mathrm{RoC}$ is capable of ablating lesions after $10 \mathrm{~s}$

11 or $30 \mathrm{~s}$ under $1.5 \mathrm{MHz}$ (full width half maximum bandwidth: $1.20-1.80 \mathrm{MHz}$ )

12 Previous studies of transcranial focused ultrasound in swine models found histologic

13 evidence of brain tissue coagulation necrosis when focal point temperatures reached $60^{\circ} \mathrm{C}$ during

$14 \quad 12-16$ s sonification or $55^{\circ} \mathrm{C}$ during 40 s sonification. ${ }^{22,23}$ Calculated thermal dose can be used to

15 predict cell death following focused energy administration. ${ }^{24,25}$ As expressed by the formula for

16 thermal dose $\left(t_{43}=\sum_{t=0}^{t=\text { final }} R^{(43-T)} \Delta t\right)$, where $\mathrm{T}$ is temperature, $\mathrm{t}$ is sonification time, and $\mathrm{R}$ is

17 related to the temperature dependence of the rate of cell death $\left(\mathrm{R}\left(\mathrm{T}<43^{\circ} \mathrm{C}\right)=1 / 4, \mathrm{R}(\mathrm{T}>43\right.$

$\left.18{ }^{\circ} \mathrm{C}\right)=1 / 2$ ), shorter sonification duration with higher thermal rise can achieve comparable thermal

19 dose to longer sonification with lower thermal rise. ${ }^{24,25}$ In the present study, measured focal point

20 temperatures reached $42^{\circ} \mathrm{C}$ at $10 \mathrm{~s}$ and $68^{\circ} \mathrm{C}$ at $30 \mathrm{~s}$, suggesting that the presented device can

21 achieve thermal ablation in brain tissue. ${ }^{23}$ Overall, these results indicate that, with further iterations

22 and miniaturizations, the device presented in this study may catalyze the development of new 
1 minimally invasive USgFUS devices, particularly those designed for use in neuro-oncological

2 settings. be enhanced.

\section{LIMITATIONS AND FUTURE DIRECTIONS}

The device reported in this study was validated using tissue-mimicking phantoms within a

12 controlled test tank, left in $23^{\circ} \mathrm{C}$ water. However, the thermal dose needed to achieve ablation is

13 known to vary by tissue type, and possessing $37^{\circ} \mathrm{C} .{ }^{25}$ As a result, additional studies using fresh

14 human brain tissue with histologic evaluation are needed to confirm successful focal point tissue

15 necrosis and the effect on surrounding cells.

Moreover, further in vivo testing is required to examine the effects of cerebrospinal fluid

17 and blood flow perfusion on focal point temperatures and surrounding tissue heating. Future

18 studies involving a larger number of array elements can also investigate electronic delays as means

19 to replace mechanical focusing of the probe to target lesions in 3D space. Finally, for the device

20 to be used from within the BrainPath ${ }^{\mathrm{TM}}$, additional studies are needed to evaluate ultrasound beam

21 refraction at the interface of the tube and the tissue. 


\section{CONCLUSION}

This study reports the development and validation of a USgFUS probe for use in MIS

3 procedures. The dimensional constraints of the prototype were designed to reflect those of

4 BrainPath ${ }^{\mathrm{TM}}$ trocars, which are MIS tools used to create atraumatic access to deep-seated brain

5 pathologies. Laboratory testing demonstrated that the MIS USgFUS prototype successfully created

6 lesions in tissue-mimicking phantoms and surpassed threshold temperatures for therapeutic applications. Real-time visualization was also achieved with a built-in imaging probe. Although this study demonstrates successful in vitro proof-of-principle, future studies should explore cadaveric validation and additional probe miniaturization for use in MIS procedures.

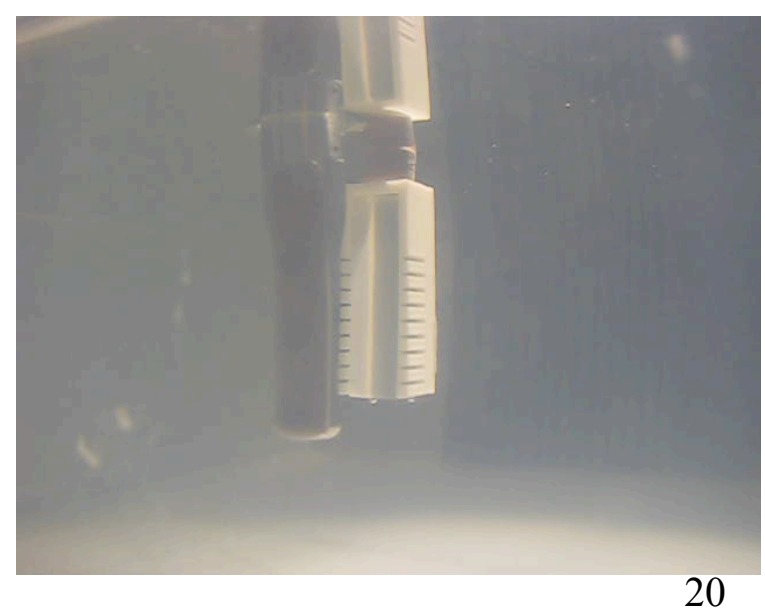

Supplementary Video 2. The video demonstrates the real-time ultrasound guidance provided by the built-in imaging probe in the device reported on this study.

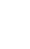

Supplementary Video 1. The video demonstrates the reported probe generating lesions in tissue-mimicking phantoms secured within a 3D-printed holder.

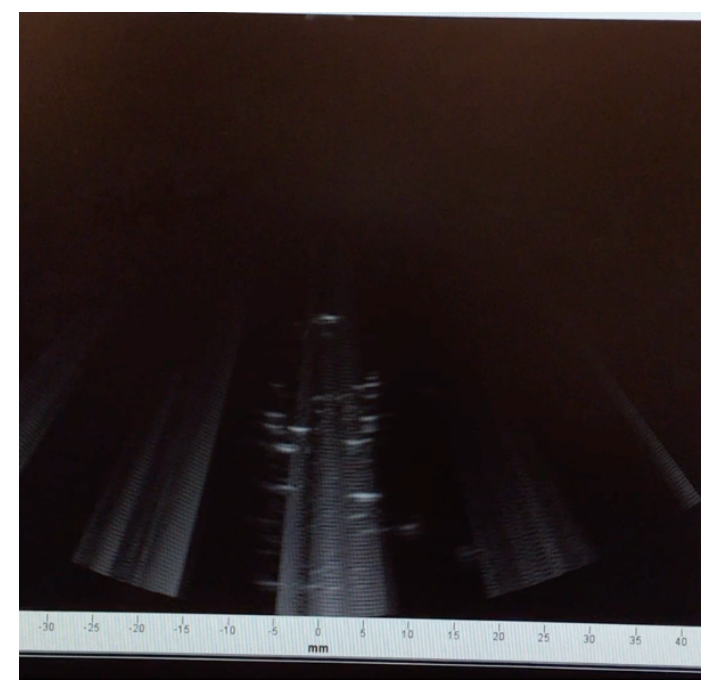




\section{REFERENCES:}

1. Escoffre J-M, Bouakaz A. Therapeutic Ultrasound. 1st ed. Springer International Publishing; 2016.

2. Cobbold RSC. Foundations of Biomedical Ultrasound. In: Foundations of Biomedical

3. McDannold N, Clement GT, Black P, Jolesz F, Hynynen K. Transcranial magnetic resonance imaging- guided focused ultrasound surgery of brain tumors: initial findings in 3 patients. Neurosurgery. 2010;66(2):323-332; discussion 332. doi:10.1227/01.NEU.0000360379.95800.2F

4. Hersh DS, Kim AJ, Winkles JA, Eisenberg HM, Woodworth GF, Frenkel V. Emerging Applications of Therapeutic Ultrasound in Neuro-oncology: Moving Beyond Tumor Ablation. Neurosurgery. 2016;79(5):643-654. doi:10.1227/NEU.0000000000001399

5. Marinova M, Strunk HM, Rauch M, et al. [High-intensity focused ultrasound (HIFU) for tumor pain relief in inoperable pancreatic cancer : Evaluation with the pain sensation scale (SES)]. Schmerz. 2017;31(1):31-39. doi:10.1007/s00482-016-0140-7

6. Magnetic Resonance-Guided Focused Ultrasound Neurosurgery for Essential Tremor: A Health Technology Assessment. Ont Health Technol Assess Ser. 2018;18(4):1-141.

7. Rumack C, Levine D. Physics of Ultrasound. In: Diagnostic Ultrasound. 5th ed. Philadelphia: Elsevier, Inc.; 2018:1-33.

8. Aubry J-F, Tanter M. MR-guided Transcranial Focused Ultrasound. In: Escoffre J-M, Bouakaz A, eds. Therapeutic Ultrasound. 1st ed. Springer; 2016:97-111.

9. Fry FJ, Barger JE. Acoustical properties of the human skull. J Acoust Soc Am. 1978;63(5):1576-1590.

10. Manbachi A, Siewerdsen J, Ellens N, et al. Flexible control and guidance of minimally invasive focused ultrasoundle. 2018.

11. Miller P. Minimally Invasive Focused Ultrasound (MIFUS) for Brain Surgery. 2018.

12. Labib MA, Abou-Al-Shaar H, Cavallo C. Minimally invasive cranial neurosurgery in the 21st century. J Neurosurg Sci. 2018;62(6):615-616. doi:10.23736/S0390-5616.18.04600-3

13. Prakash P, Salgaonkar VA, Diederich CJ. Modelling of endoluminal and interstitial ultrasound hyperthermia and thermal ablation: applications for device design, feedback control and treatment planning. Int J Hyperth Off J Eur Soc Hyperthermic Oncol North Am Hyperth Gr. 2013;29(4):296-307. doi:10.3109/02656736.2013.800998

14. Fleury G, Berriet R, Chupin L, et al. High intensity therapeutic ultrasound transducer performance and characterisation. In: 2010 IEEE International Ultrasonics Symposium. San Diego; 2010:876-885. doi:10.1109/ULTSYM.2010.5935910

15. Cohen AR. Pediatric Neurosurgery: Tricks of the Trade. Thieme; 2015.

16. Minimally Invasive Neuroendoscopy. Johns Hopkins Medicine. https://www.hopkinsmedicine.org/neurology_neurosurgery/centers_clinics/brain_tumor/tr eatment/surgery/neuroendoscopy.html. Published 2019.

17. Sussman ES, Kellner CP, Nelson E, et al. Hemorrhagic complications of ventriculostomy: incidence and predictors in patients with intracerebral hemorrhage. J Neurosurg. 2014;120(4):931-936. doi:10.3171/2013.12.JNS131685

18. Chowdhry SA, Cohen AR. Intraventricular neuroendoscopy: complication avoidance and management. World Neurosurg. 2013;79(2 Supp1):S15.e1-10. doi:10.1016/j.wneu.2012.02.030

19. Belzberg M, Chavez F, Xiong KT, et al. Minimally invasive intraventricular ultrasound: 
design and instrumentation towards a miniaturized ultrasound-guided focused ultrasound probe. Proc SPIE 10951, Med Imaging 2019 Image-Guided Proced Robot Interv Model. 2019;109512Y.

20. Zhang X, Ellens N, Belzberg M, et al. Design of a Low-power, Minimally Invasive High Intensity Focused Ultrasound Device for Ablative Applications in Neuro-Oncology: A Simulation Study. In: 59th AAPM (American Association of Physicists in Medicine) Annual Meeting \& Exhibition. Denver; 2017.

21. Jung Na Young Rachmilevitch Itay SOATZECJW. Factors Related to Successful Energy Transmission of Focused Ultrasound through a Skull : A Study in Human Cadavers and Its Comparison with Clinical Experiences. J Korean Neurosurg Soc. 0. doi:10.3340/jkns.2018.0226

22. Cohen ZR, Zaubermann J, Harnof S, et al. Magnetic Resonance Imaging-Guided Focused Ultrasound for Thermal Ablation in the Brain. Neurosurgery. 2007;60(4):593-600. doi:10.1227/01.neu.0000245606.99946.c6

23. $\mathrm{Xu} \mathrm{Z,} \mathrm{Carlson} \mathrm{C,} \mathrm{Snell} \mathrm{J,} \mathrm{et} \mathrm{al.} \mathrm{Intracranial} \mathrm{inertial} \mathrm{cavitation} \mathrm{threshold} \mathrm{and} \mathrm{thermal}$ ablation lesion creation using MRI-guided 220-kHz focused ultrasound surgery: preclinical investigation. J Neurosurg. 2014;122(1):152-161. doi:10.3171/2014.9.jns 14541

24. Sapareto SA, Dewey WC. Thermal dose determination in cancer therapy. Int J Radiat Oncol Biol Phys. 1984;10(6):787-800.

25. Van Rhoon GC, Samaras T, Yarmolenko PS, Dewhirst MW, Neufeld E, Kuster N. $\mathrm{CEM} 43^{\circ} \mathrm{C}$ thermal dose thresholds: A potential guide for magnetic resonance radiofrequency exposure levels? Eur Radiol. 2013;23(8):2215-2227. doi:10.1007/s00330$013-2825-\mathrm{y}$ 